\title{
Low Preoperative Serum Albumin Levels Are Associated With Impaired Outcome After Cytoreductive Surgery and Perioperative Intraperitoneal Chemotherapy for Peritoneal Surface Malignancies
}

\author{
Charalampos Seretis ${ }^{\mathrm{a}, \mathrm{c}}$, Jagjit Gill ${ }^{\mathrm{a}}$, Adnan Malik ${ }^{\mathrm{a}}$, Ali Mohamed Elhassan ${ }^{\mathrm{a}}$, \\ Umar Shariff $^{b}$, Haney Youssef ${ }^{b}$
}

\begin{abstract}
Preoperative hypoalbuminemia is known to be associated with postoperative morbidity and mortality, as well as with poor survival after gastrointestinal cancer surgery. However, limited data exist regarding the prognostic significance of hypoalbuminemia in patients with peritoneal metastases undergoing cytoreductive surgery, combined with perioperative intraperitoneal chemotherapy. We performed a systematic literature review of the previously published studies addressing the potential association between preoperative albumin levels and overall surgical outcomes after the performance of cytoreductive surgery and perioperative intraperitoneal chemotherapy for peritoneal surface malignancies. Our research yielded a total of nine retrospective studies which met our inclusion criteria, and despite their heterogeneity; and we can conclude that preoperatively low albumin levels are associated with greater likelihood of overall and major morbidity, as well as less favorable oncological outcome after the performance of cytoreductive surgery and perioperative intraperitoneal chemotherapy.
\end{abstract}

Keywords: Albumin; Cytoreductive surgery; Intraperitoneal chemotherapy; HIPEC; Nutrition; Cancer; Peritoneal malignancy

\section{Introduction}

Cytoreductive surgery (CRS) combined with perioperative intraperitoneal chemotherapy (PIC) is considered a promising

Manuscript submitted September 27, 2020, accepted October 9, 2020

Published online December 18, 2020

${ }^{a}$ George Eliot Hospital NHS Trust, Warwickshire, UK

${ }^{\mathrm{b}}$ General Surgery Department, Good Hope Hospital, Heart of England NHS Foundation Trust, Birmingham, UK

${ }^{\mathrm{c} C o r r e s p o n d i n g ~ A u t h o r: ~ C h a r a l a m p o s ~ S e r e t i s, ~ S p e c i a l t y ~ R e g i s t r a r ~ i n ~ G e n e r a l ~}$ Surgery, George Eliot Hospital NHS Trust, College Street, CV10 7DJ, Warwickshire, UK. Email: charalampos.seretis@geh.nhs.uk

doi: https://doi.org/10.14740/jocmr4362 treatment option in appropriately selected patients with peritoneal metastatic disease and primary peritoneal malignancies $[1,2]$. CRS aims to achieve complete macroscopic removal of the visible cancerous deposits within the abdominal cavity (complete cytoreduction), while the administration of PIC is used to achieve microscopic disease clearance, targeting the intra-abdominally circulating cancer cells and cancerous lesions of few cell lines depth [3]. Due to the nature of the operation, which usually requires multi-visceral resections, and the adverse effects of the intraperitoneal chemotherapy, this combined procedure carries considerable postoperative morbidity and mortality, even in high-volume centers [4]. In order to improve the postoperative outcome and the oncological benefit of this complex procedure, several key areas for improvement have been identified over the last years. These include the recognition of the role mentorship and senior supervision to overcome the procedure's steep learning curve [5-7], the rationalization of patient selection criteria for CRS and PIC [8,9], the improvement of imaging techniques used to guide the operative decision-making [10], along with efforts to reduce the toxicity of the intraperitoneal chemotherapy and optimize the postoperative care of these patients [11-13].

Interestingly, there is a relative lack of studies focusing on the impact of malnutrition on postoperative morbidity and mortality, as well as the survival after CRS and PIC. The latter is of great importance given the widely accepted value of optimizing the patients' nutrition status prior to any major abdominal procedure, which has been shown to be inversely correlated with overall complication rates and length of hospitalization $[14,15]$. Moreover, particularly in patients undergoing major abdominal oncological operation, preoperative malnutrition has been strongly associated with poorer survival $[16,17]$. Therefore, it is reasonable to assume that preoperative detection of patients at nutritional risk and enhanced nutritional support could help to achieve a reduction of morbidity and mortality after CRS and PIC, and potentially improve the oncological outcomes of the procedure. Under this notion, the preoperative evaluation of widely available malnutrition markers, with serum albumin being the traditional laboratory reference test [18] could be of value in the planning of 

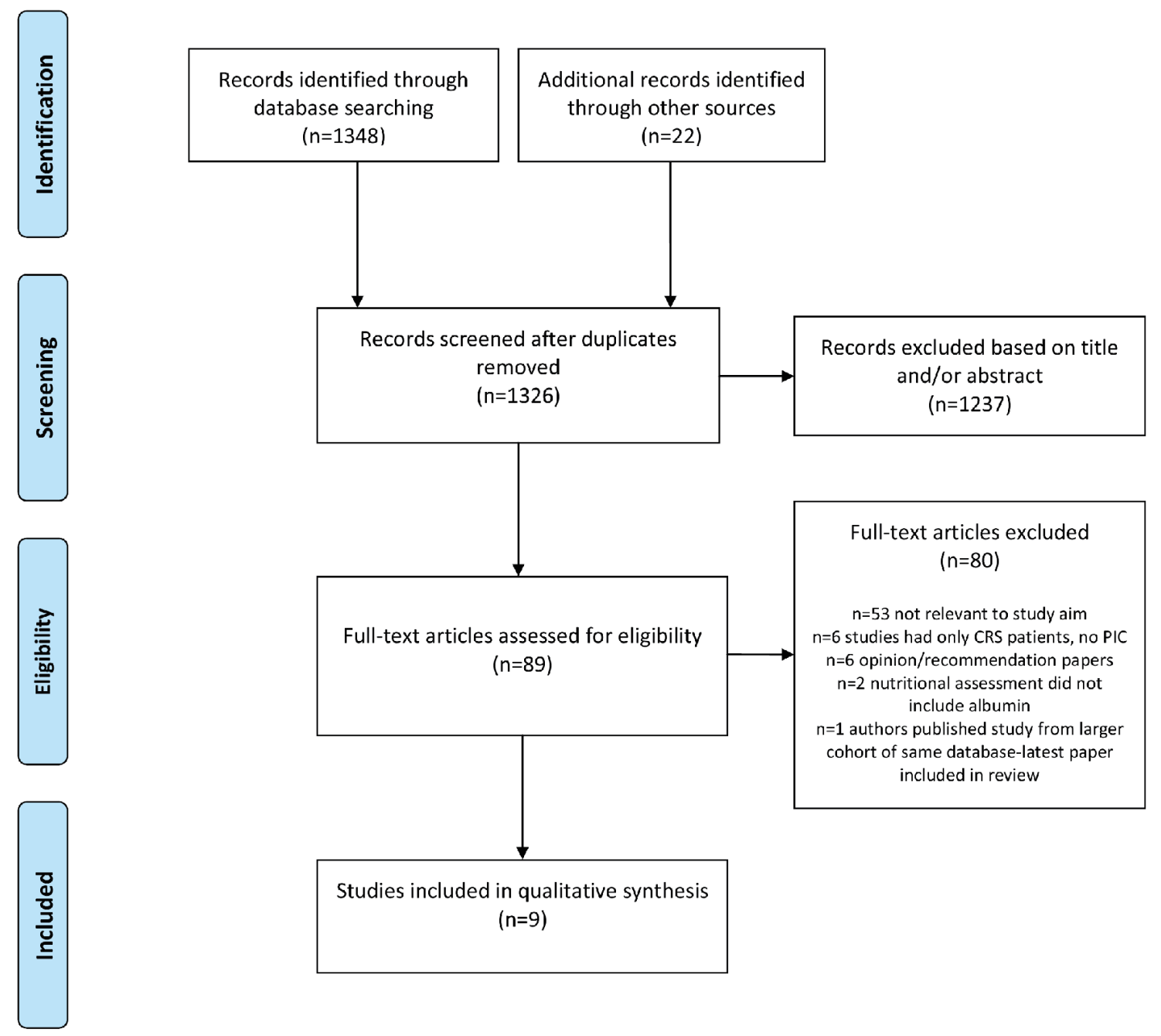

Figure 1. Flowchart of the systematic review.

a holistic treatment plan. To address this question, we have performed a systematic review of the literature of the studies examining an association of preoperative albumin levels with the occurrence of postoperative morbidity and mortality, as well as survival after CRS and PIC for peritoneal surface malignancies.

\section{Methods}

We performed a systematic review of the studies indexed in PubMed database until August 2020, using the search terms ("albumin" AND "cytoreductive surgery") and ("albumin" AND "intraperitoneal chemotherapy"), in accordance to PRISMA guidelines. Citations of articles retrieved at the initial literature search were manually searched to identify further relevant studies meeting the purpose and selection criteria of this systematic review. We excluded studies referring to pediatric population, as well as studies where CRS was performed but not followed by PIC. Also, we excluded studies published in other than English language as well as case reports and case series.

\section{Results}

A total of nine studies [19-27], all retrospective in nature, met our inclusion criteria (Fig. 1). Six out of the nine included studies included patients with peritoneal disease arising from two or more primary origins [19-23, 25], while two studies used as study population patients of older age groups, who had undergone CRS and PIC [20, 25]. Despite the significant heterogeneity in terms of primary malignancies, extent of peritoneal disease and differences in patient selection and treatment protocols, the main two clinical questions of our systematic review, association of albumin levels with postoperative morbidity/mortality and survival after CRS and PIC, could be answered in a clear manner.

With respect to the potential value of preoperative serum albumin as a prognostic factor of survival after CRS and PIC, seven of the studies addressed this point [19, 21, 23-27]. In 
five of those studies, preoperatively lower albumin levels, as defined in each study by the authors, were strongly associated with poorer survival rates [19, 21, 23, 24, 26, 27], while Votanopoulos and co-workers [25] did not demonstrate any relevant prognostic significance of preoperative albumin in their cohort of patients after CRS and PIC. The key features of the included studies (study population, origin of peritoneal disease, postoperative morbidity and mortality, as well as survival outcomes) and information regarding the correlations of preoperative serum albumin levels with the operative and oncological outcome are presented in detailed manner in Table 1 [19-27].

\section{Discussion}

Serum albumin has traditionally been considered as a classical laboratory marker reflecting surgical patients' nutritional status, despite the fact that its levels can be influenced by a variety of factors (i.e., acute illness, chronic inflammatory response, and liver pathologies, etc) [28, 29]. However, the low cost of the relevant laboratory essays and the wide availability of this examination as a basic paraclinical parameter, has led to its disseminated use as a nutritional marker in the general surgery practice [30]. The latter is of great importance in surgical oncology, as apart from the surgical stress and the subsequent catabolic cascade, the operable patients can frequently be in preclinical stages of cancer-related cachexia with evolving skeletal muscle depletion $[31,32]$. Therefore, preoperative serum albumin levels have been highlighted by numerous previous studies in the field of surgical oncology as an important marker of perioperative morbidity and survival after surgery for gastrointestinal malignancies [33, 34].

Taking into consideration the greater magnitude of cancer burden in cases of peritoneal metastatic disease, which very frequently can present with symptoms of gastrointestinal intolerance and poor oral intake, the importance of adequate nutritional status is a key feature for the success of a major procedure as CRS and PIC. Previous studies in patients with peritoneal malignancy undergoing CRS and PIC have clearly demonstrated the postoperative occurrence of prolonged ileus, which prohibits early oral nutrition; even early enteral nutrition has been shown not to be well tolerated in the immediate postoperative phase, requiring frequently the postoperative administration of parenteral nutrition [35]. Furthermore, the major stress assault from this extensive surgical procedure results in a significant drop of postoperative serum albumin levels, indicating the extent of the post-surgical inflammatory response and the initiation of a catabolic response [36]. As a result, it is evident that patients scheduled for CRS and PIC need to be nutritionally optimized before embarking for surgery with a structured specialist nutritional assessment and supplementation as required, and serum albumin should be included as a baseline laboratory marker in the nutrition enhancement pathway.

Although the above-mentioned seem reasonable, very few studies [19-27] have previously assessed the usefulness of preoperative albumin levels as a marker of post-CRS and PIC morbidity and/or survival outcomes. Our study is the first in the literature to review in a systematic manner the available evi- dence of this topic. Despite their heterogeneity with regards to primary cancer origin, extent of peritoneal metastatic disease and different albumin cut-off values, the overall qualitative conclusion from our review study is that preoperatively low albumin levels are associated with an increase in postoperative morbidity, especially grade III/IV complications, and have a prognostic value as a marker of survival post CRS and PIC. As presented in the Table 1 summarizing the relevant findings of the included studies, there is no universal agreement to define a cut-off value of serum albumin value as a predictor of expected postoperative morbidity and/or mortality. However, the majority of included studies define hypoalbuminemia when serum albumin value was lower than $3.5 \mathrm{~g} / \mathrm{dL}$, and therefore this value potentially could be a starting point for future prospectively conducted studies, as a target to reach before proceeding with CRS and PIC. Considering the known limitations of albumin as the sole laboratory marker of nutritional status, effort should be made towards the formation of nutritional risk stratification scoring systems, combining laboratory markers (albumin, pre-albumin, electrolytes and minerals, etc.) with anthropometric nutritional assessments (body mass index (BMI), skinfold thickness, mid-arm muscle circumference, etc.), existing validated nutritional risk tools (MUST, SNAQ, MNA-SF, etc.) and perhaps imaging techniques, such as estimation of skeletal mass via the standard preoperative computed tomography. We believe that preoperative enhanced nutrition should be considered as a standard parameter of the preoperative assessment and patient optimization before CRS with PIC, and serum albumin levels should be assessed as a standard parameter in the preoperative workup of these patients.

\section{Acknowledgments}

None to declare.

\section{Financial Disclosure}

None to declare.

\section{Conflict of Interest}

None to declare.

\section{Author Contributions}

$\mathrm{CS}, \mathrm{AM}, \mathrm{JG}$ and AME equally contributed to the performance of literature review and drafting of the manuscript; US and HY contributed to the critical revision of the manuscript, which all listed co-authors approve in its final format.

\section{Data Availability}

The authors declare that data supporting the findings of this 


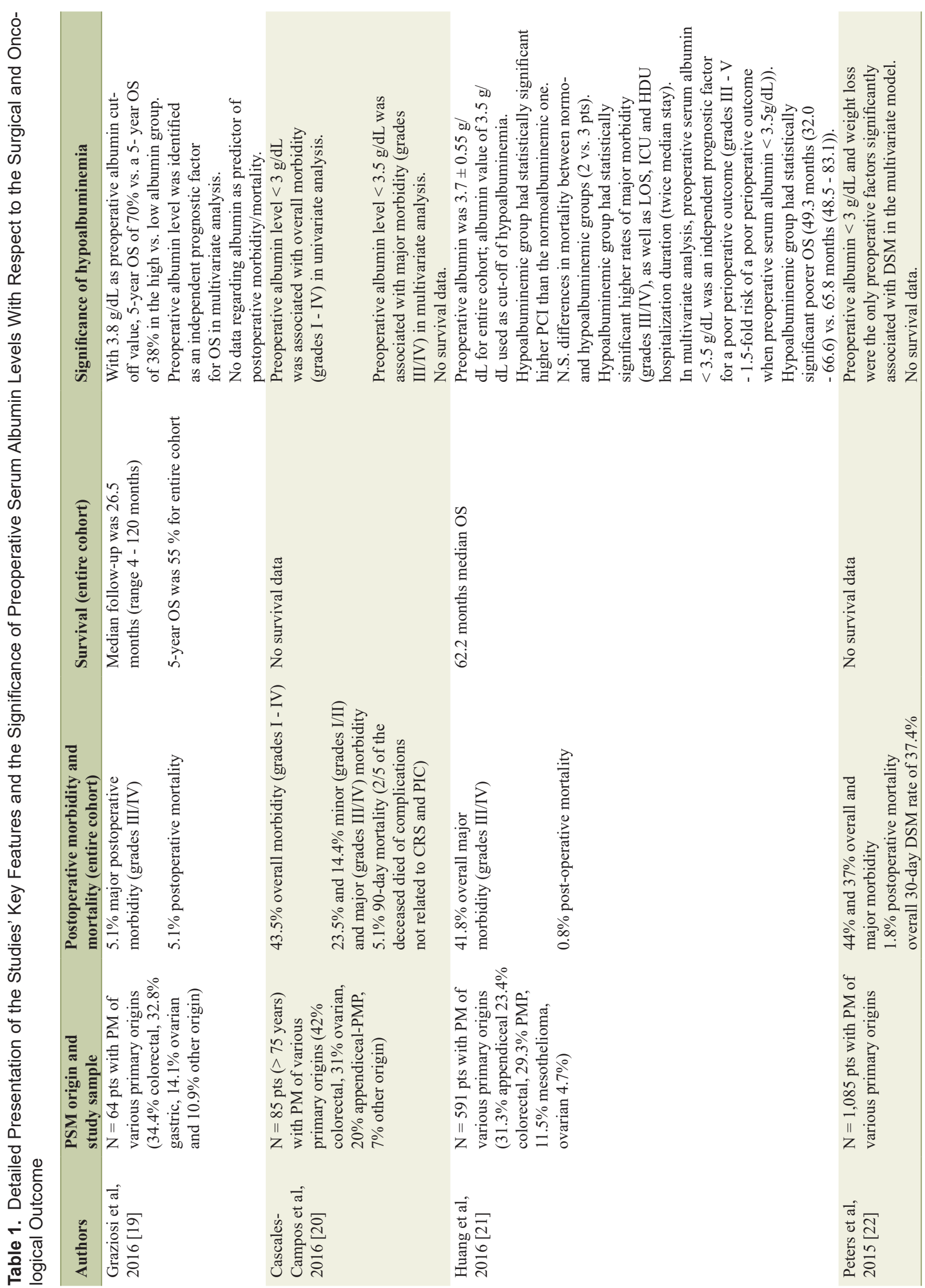




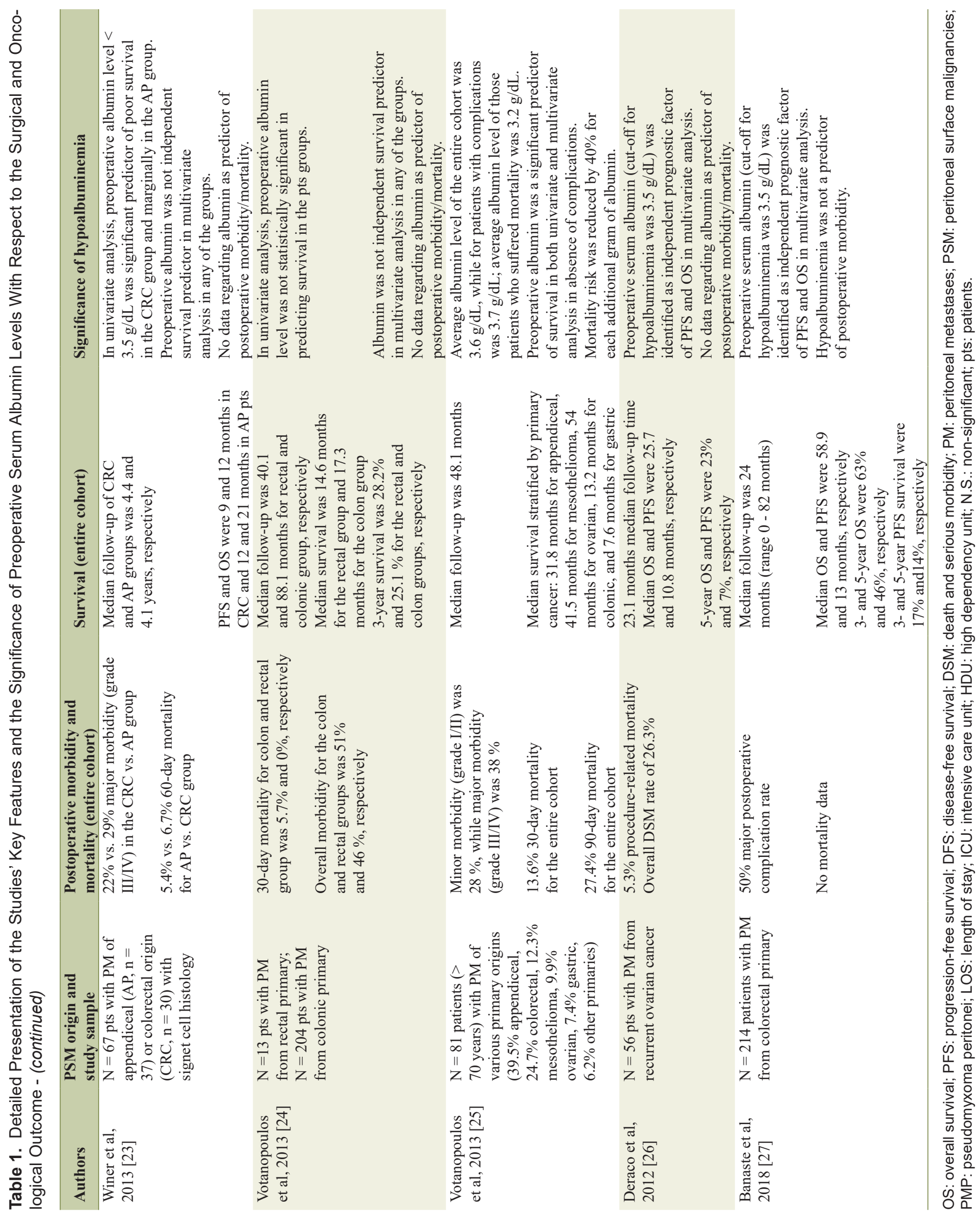


study are available within the article.

\section{References}

1. Sugarbaker PH. Cytoreductive surgery and hyperthermic intraperitoneal chemotherapy in the management of gastrointestinal cancers with peritoneal metastases: Progress toward a new standard of care. Cancer Treat Rev. 2016;48:42-49.

2. Mohamed F, Cecil T, Moran B, Sugarbaker P. A new standard of care for the management of peritoneal surface malignancy. Curr Oncol. 2011;18(2):e84-96.

3. Sugarbaker PH. Management of peritoneal metastases Basic concepts. J BUON. 2015;20(Suppl 1):S2-11.

4. Chua TC, Yan TD, Saxena A, Morris DL. Should the treatment of peritoneal carcinomatosis by cytoreductive surgery and hyperthermic intraperitoneal chemotherapy still be regarded as a highly morbid procedure?: a systematic review of morbidity and mortality. Ann Surg. 2009;249(6):900-907.

5. Kusamura S, Moran BJ, Sugarbaker PH, Levine EA, Elias D, Baratti D, Morris DL, et al. Multicentre study of the learning curve and surgical performance of cytoreductive surgery with intraperitoneal chemotherapy for pseudomyxoma peritonei. Br J Surg. 2014;101(13):1758-1765.

6. Voron T, Eveno C, Jouvin I, Beaugerie A, Lo Dico R, Dagois S, Soyer P, et al. Cytoreductive surgery with a hyperthermic intraperitoneal chemotherapy program: Safe after 40 cases, but only controlled after 140 cases. Eur J Surg Oncol. 2015;41(12):1671-1677.

7. Mohamed F, Moran BJ. Morbidity and mortality with cytoreductive surgery and intraperitoneal chemotherapy: the importance of a learning curve. Cancer J. 2009;15(3):196199.

8. Esquivel J. Cytoreductive surgery and hyperthermic intraperitoneal chemotherapy for colorectal cancer: survival outcomes and patient selection. J Gastrointest Oncol. 2016;7(1):72-78.

9. Riss S, Mohamed F, Dayal S, Cecil T, Stift A, Bachleitner-Hofmann T, Moran B. Peritoneal metastases from colorectal cancer: patient selection for cytoreductive surgery and hyperthermic intraperitoneal chemotherapy. Eur J Surg Oncol. 2013;39(9):931-937.

10. Torkzad MR, Casta N, Bergman A, Ahlstrom H, Pahlman L, Mahteme H. Comparison between MRI and CT in prediction of peritoneal carcinomatosis index (PCI) in patients undergoing cytoreductive surgery in relation to the experience of the radiologist. J Surg Oncol. 2015;111(6):746-751.

11. Kusamura S, Baratti D, Younan R, Laterza B, Oliva GD, Costanzo P, Favaro M, et al. Impact of cytoreductive surgery and hyperthermic intraperitoneal chemotherapy on systemic toxicity. Ann Surg Oncol. 2007;14(9):25502558.

12. Webb CA, Weyker PD, Moitra VK, Raker RK. An overview of cytoreductive surgery and hyperthermic intraperitoneal chemoperfusion for the anesthesiologist. Anesth Analg. 2013;116(4):924-931.
13. Owusu-Agyemang P, Soliz J, Hayes-Jordan A, Harun N, Gottumukkala V. Safety of epidural analgesia in the perioperative care of patients undergoing cytoreductive surgery with hyperthermic intraperitoneal chemotherapy. Ann Surg Oncol. 2014;21(5):1487-1493.

14. Cerantola Y, Grass F, Cristaudi A, Demartines N, Schafer M, Hubner M. Perioperative nutrition in abdominal surgery: recommendations and reality. Gastroenterol Res Pract. 2011;2011:739347.

15. Hu WH, Cajas-Monson LC, Eisenstein S, Parry L, Cosman B, Ramamoorthy S. Preoperative malnutrition assessments as predictors of postoperative mortality and morbidity in colorectal cancer: an analysis of ACS-NSQIP. Nutr J. 2015;14:91.

16. Benoist $\mathrm{S}$, Brouquet A. Nutritional assessment and screening for malnutrition. J Visc Surg. 2015;152(Suppl 1):S3-7.

17. Tokunaga R, Sakamoto Y, Nakagawa S, Miyamoto Y, Yoshida N, Oki E, Watanabe M, et al. Prognostic nutritional index predicts severe complications, recurrence, and poor prognosis in patients with colorectal cancer undergoing primary tumor resection. Dis Colon Rectum. 2015;58(11):1048-1057.

18. Gupta D, Lis CG. Pretreatment serum albumin as a predictor of cancer survival: a systematic review of the epidemiological literature. Nutr J. 2010;9:69.

19. Graziosi L, Marino E, De Angelis V, Rebonato A, Donini A. Survival prognostic factors in patients undergoing cytoreductive surgery and hyperthermic intraperitoneal chemotherapy treatment: analysis from a single oncological center. World J Surg Oncol. 2016;14:97.

20. Cascales-Campos PA, Lopez-Lopez V, Munoz-Casares FC, Feliciangeli E, Torres Melero J, Barrios P, Morales $\mathrm{R}$, et al. Morbidity and mortality outcomes after cytoreductive surgery and hyperthermic intraperitoneal chemotherapy in patients aged 75 years and over: Spanish group of peritoneal cancer surgery (GECOP) multicenter study. Surg Oncol. 2016;25(2):111-116.

21. Huang Y, Alzahrani NA, Chua TC, Huo YR, Liauw W, Morris DL. Impacts of preoperative serum albumin level on outcomes of cytoreductive surgery and perioperative intraperitoneal chemotherapy. Ann Surg Oncol. 2016;23(8):2411-2418.

22. Peters MG, Bartlett EK, Roses RE, Kelz RR, Fraker DL, Karakousis GC. Age-related morbidity and mortality with cytoreductive surgery. Ann Surg Oncol. 2015;22(Suppl 3):S898-904.

23. Winer J, Zenati M, Ramalingam L, Jones H, Zureikat A, Holtzman M, Lee K, et al. Impact of aggressive histology and location of primary tumor on the efficacy of surgical therapy for peritoneal carcinomatosis of colorectal origin. Ann Surg Oncol. 2014;21(5):1456-1462.

24. Votanopoulos KI, Swett K, Blackham AU, Ihemelandu C, Shen P, Stewart JH, Levine EA. Cytoreductive surgery with hyperthermic intraperitoneal chemotherapy in peritoneal carcinomatosis from rectal cancer. Ann Surg Oncol. 2013;20(4):1088-1092.

25. Votanopoulos KI, Newman NA, Russell G, Ihemelandu C, Shen P, Stewart JH, Levine EA. Outcomes of Cytore- 
ductive Surgery (CRS) with hyperthermic intraperitoneal chemotherapy (HIPEC) in patients older than 70 years; survival benefit at considerable morbidity and mortality. Ann Surg Oncol. 2013;20(11):3497-3503.

26. Deraco M, Virzi S, Iusco DR, Puccio F, Macri A, Famulari C, Solazzo M, et al. Secondary cytoreductive surgery and hyperthermic intraperitoneal chemotherapy for recurrent epithelial ovarian cancer: a multi-institutional study. BJOG. 2012;119(7):800-809.

27. Banaste N, Rousset P, Mercier F, Rieussec C, Valette PJ, Glehen O, Passot G. Preoperative nutritional risk assessment in patients undergoing cytoreductive surgery plus hyperthermic intraperitoneal chemotherapy for colorectal carcinomatosis. Int J Hyperthermia. 2018;34(5):589-594.

28. Doweiko JP, Nompleggi DJ. Role of albumin in human physiology and pathophysiology. JPEN J Parenter Enteral Nutr. 1991;15(2):207-211.

29. Klein $\mathrm{S}$. The myth of serum albumin as a measure of nutritional status. Gastroenterology. 1990;99(6):1845-1846.

30. Seres DS. Surrogate nutrition markers, malnutrition, and adequacy of nutrition support. Nutr Clin Pract. 2005;20(3):308-313.

31. Hubner M, Mantziari S, Demartines N, Pralong F, Coti-Bertrand P, Schafer M. Postoperative albumin drop is a marker for surgical stress and a predictor for clini- cal outcome: a pilot study. Gastroenterol Res Pract. 2016;2016:8743187.

32. Fearon KC, Voss AC, Hustead DS, Cancer Cachexia Study G. Definition of cancer cachexia: effect of weight loss, reduced food intake, and systemic inflammation on functional status and prognosis. Am J Clin Nutr. 2006;83(6):1345-1350.

33. Moghadamyeghaneh Z, Hwang G, Hanna MH, Phelan MJ, Carmichael JC, Mills SD, Pigazzi A, et al. Even modest hypoalbuminemia affects outcomes of colorectal surgery patients. Am J Surg. 2015;210(2):276-284.

34. Lai CC, You JF, Yeh CY, Chen JS, Tang R, Wang JY, Chin CC. Low preoperative serum albumin in colon cancer: a risk factor for poor outcome. Int J Colorectal Dis. 2011;26(4):473-481.

35. Dineen SP, Robinson KA, Roland CL, Beaty KA, Rafeeq S, Mansfield PF, Royal RE, et al. Feeding tube placement during cytoreductive surgery and heated intraperitoneal chemotherapy does not improve postoperative nutrition and is associated with longer length of stay and higher readmission rates. J Surg Res. 2016;200(1):158-163.

36. Cooksley TJ, Haji-Michael P. Post-operative critical care management of patients undergoing cytoreductive surgery and heated intraperitoneal chemotherapy (HIPEC). World J Surg Oncol. 2011;9:169. 